\title{
SELF-MICRO EMULSIFYING DRUG DELIVERY SYSTEMS (SMEDDS): A REVIEW ON PHYSICO-CHEMICAL AND BIOPHARMACEUTICAL ASPECTS
}

\author{
Sheo Datta Maurya ${ }^{* 1}$, Rajeshwar K.K. Arya ${ }^{2}$, G. Rajpal ${ }^{2}$, Ram C. Dhakar ${ }^{3}$ \\ ${ }^{1}$ Department of Pharmacy, IEC Group of Institution, Greater Noida, India-201306 \\ ${ }^{2}$ Department of Pharmaceutical Sciences, Kumaun University, Bhimtal, Nainital, UK, India-263136 \\ ${ }^{3}$ Jhalawar Medical College and Hospital, N.H.-12, Jhalawar, Rajasthan, India-326001
}

Article Info: Received 02 April 2017; Review Completed 13 May 2017; Accepted 13 May 2017, Available online 15 May 2017

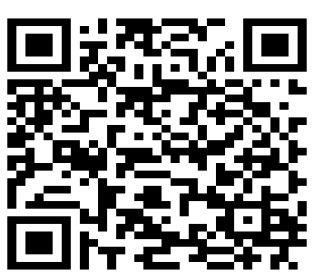

Cite this article as:

Maurya SD, Arya RKK, Rajpal G, Dhakar RC, Self-micro emulsifying drug delivery systems (SMEDDS): a review on physico-chemical and biopharmaceutical aspects, Journal of Drug Delivery and Therapeutics. 2017; 7(3):55-65 DOI: http://dx.doi.org/10.22270/jddt.v7i3.1453

*Address for Correspondence:

Sheo Datta Maurya, Department of Pharmacy, IEC Group of Institution, Greater Noida, India-201306 Email: meetshiv23@gmail.com

\begin{abstract}
Nearly $40 \%$ of new drug candidates exhibit low solubility in water, which is a challenge in development of optimum oral solid dosage form in terms of formulation design and bioavailability of new pharmaceutical products. Many strategies have been used to overcome these problems either by means of modifying the solubility or maintaining the drug in dissolved form throughout gastric transit time. Much attention has focused on lipid solutions, emulsions and emulsion pre-concentrates, which can be prepared as physically stable formulations suitable for encapsulation of such poorly soluble drugs. Recently, self-micro emulsifying drug delivery systems (SMEDDS) especially have attracted increasing interest primarily because these are physically stable, easy to manufacture, can be filled in soft gelatin capsules and then will generate a drug containing micro-emulsion with a large surface area upon dispersion in the gastrointestinal tract. The emulsions will further facilitate the absorption of the drug due via intestinal lymphatic pathway and by partitioning of drug into the aqueous phase of intestinal fluids. In the present review, an overview of SMEDDS as a key technology for formulating lipophilic drugs and various factors that potentially affect the oral bioavailability of such drugs are presented.
\end{abstract}

Keywords: Low solubility, Oral bioavailability, Self-micro emulsion, Intestinal lymphatic pathway.

\section{INTRODUCTION}

Various techniques are used to enhance oral bioavailability of poorly water soluble drugs ${ }^{1,2,3}$. Oral route has been the major route of drug delivery for the chronic treatment of many diseases as it offers a high degree of patient compliance. However, oral delivery of $50 \%$ of the drug compounds is hampered because of the high lipophilicity of the drug itself. Nearly $40 \%$ of new drug candidates exhibit low solubility in water, which is a challenge in development of optimum oral solid dosage form in terms of formulation design and bioavailability of new pharmaceutical products. Many strategies have been used to overcome these problems either by means of modifying the solubility or maintaining the drug in dissolved form throughout gastric transit time ${ }^{4,5}$. These strategies may include the use of surfactants, cyclodextrins, micronization, liquisolid techniques ${ }^{6}$, salt formation, $\mathrm{pH}$ change, nano size delivery ${ }^{7}$, solid dispersions ${ }^{8,9}$ and permeation enhancers and ${ }^{10},{ }^{11}$.

Much attention has focused on lipid solutions, emulsions and emulsion preconcentrates, which can be prepared as physically stable formulations suitable for encapsulation of such poorly soluble drugs. Emulsion systems are associated with their own set of complexities, including stability and manufacturing problems associated with their commercial production. Self-emulsification systems are one formulation technique that can be a fitting answer to such problems ${ }^{12}$. 
Among the lipid-based systems, Self-emulsifying drug delivery systems (SEDDS) is a promising strategy to improve the bioavailability of poorly water-soluble compounds. SEDDS are isotropic mixtures of drug, lipids and surfactants, usually with one or more hydrophilic co-solvents or co-emulsifiers ${ }^{13}$. Upon mild agitation followed by dilution with aqueous media, these systems can form fine (oil in water) emulsion instantaneously. The size of the droplet formed is between 100 and $300 \mathrm{~nm}$ while self-micro-emulsifying drug delivery systems (SMEDDS) form transparent micro-emulsions with a droplet size of less than $50 \mathrm{~nm}$ 14

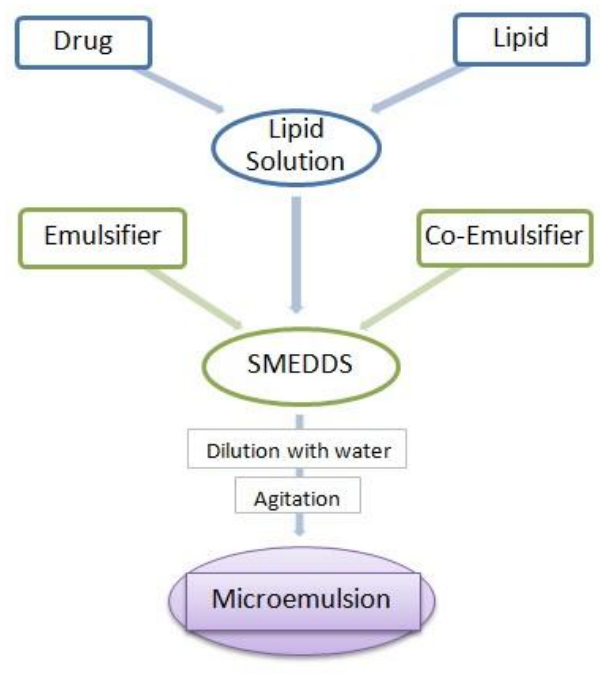

Figure 1: The general strategy of formulating SMEDDS and their subsequent conversion to micro-emulsion

In self emulsifying formulations, the formed emulsion increases membrane permeability as a result of surfactant presence and enhances lymphatic absorption (lymphatic transport) due to medium and long chain oils. These factors may contribute significantly to the better performance of the formulations ${ }^{15}, 16,17,18$.

Recently, SMEDDS especially have attracted increasing interest primarily because SMEDDS are physically stable, easy to manufacture, can be filled in soft gelatin capsules and then will generate a drug containing microemulsion with a large surface area upon dispersion in the gastrointestinal tract. The emulsions will further facilitate the absorption of the drug due to a faster digestion by gastrointestinal enzymes and subsequent transfer to mixed micelles or possible absorption directly from the emulsion particle, by partitioning of drug into the aqueous phase of intestinal fluids. ${ }^{19}$ Herein, an overview of SMEDDS as a key technology for formulating lipophilic drugs and increasing their oral bioavailability is presented.

Table 1: Application of SMEDDS in various BCS class drugs ${ }^{25}$

\begin{tabular}{|l|l|l|l|}
\hline BCS class & $\begin{array}{l}\text { Aqueous } \\
\text { Solubility }\end{array}$ & $\begin{array}{l}\text { Membrane } \\
\text { permeability }\end{array}$ & Problems \\
\hline Class I & High & High & Enzymatic degradation, gut wall efflux \\
\hline Class II & Low & High & Solubilization and bioavailability \\
\hline Class III & High & Low & Enzymatic degradation, gut wall efflux and bioavailability \\
\hline Class IV & Low & Low & Solubilization, enzymatic degradation, gut wall efflux and bioavailability \\
\hline
\end{tabular}

\section{PHYSICO-CHEMICAL ASPECTS OF SMEDDS}

\subsection{Formulation/ Composition of SMEDDS}

The formulation generally consists of drug, oily vehicle, surfactant, co-surfactant and even co-solvents. The basic principle of this system is its ability to form fine oil-inwater $(\mathrm{o} / \mathrm{w})$ micro-emulsions under gentle agitation following dilution by aqueous phases (ie, the digestive motility of the stomach and intestine provide the agitation required for self-emulsification in vivo in the lumen of the gut). ${ }^{20}$ This spontaneous formation of an emulsion in the gastrointestinal tract presents the drug in a solubilized form, and the small size of the formed droplet provides a large interfacial surface area for drug absorption. ${ }^{21}$ Apart from solubilization, the presence of lipid in the formulation further helps improve bioavailability by affecting the drug absorption. ${ }^{22}$ Selection of a suitable self-emulsifying formulation depends upon the assessment of (1) physicochemical properties of the drug, such as $\mathrm{pKa}$, polarity and solubility in various components (2) physicochemical nature of oily phase, surfactant and co-surfactant (3) the area of the self-emulsifying region as obtained in the phase diagram, (4) the ratio of the components, especially oil to surfactant ratio and (5) the droplet size distribution of the resultant emulsion following selfemulsification. ${ }^{23}$

\subsubsection{Selection of drug for SMEDDS}

It is important to know that the drug of interest can also have significant impact on the various aspects of SMEDDS, such as phase behavior and micro-emulsion droplet size. Various physicochemical properties of the drug, such as $\mathrm{pKa}, \log \mathrm{P}$, molecular structure and weight, presence of ionizable groups and the quantity have considerable effects on the performance of SMEDDS. Drugs those have low therapeutic dose are ideal drug candidates for SMEDDS. ${ }^{14}$

One of the primary challenges in designing of oral formulation is maintaining drug solubility within the G.I.T. and, in particular, maximizing drug solubility within the prime absorptive site of the gut ${ }^{24}$. Drugs which are administered at very high dose are not suitable for SMEDDS unless they have good solubilization in at least one of the excipients of SMEDDS, preferably lipophillic phase. The drug must be physically and chemically stable in the formulation and the drug release pattern must remain constant during the shelf life of the SMEDDS formulation. These systems can help in solving the under-mentioned problems of all the categories of BCS class drugs, as depicted in Table 1. 


\subsubsection{Selection of Excipients for SMEDDS}

The excipients should be chosen from the list of generally regarded as safe "GRAS" excipients published by USFDA. Good understanding of the characteristics of excipients and their performance in formulations are the necessary requirements for successful formulation development. In order to formulate a successful SMEDDS for maximum therapeutic effect, due consideration must be given to following factors;

$\checkmark$ Physicochemical properties of the drug as well as excipients

$\checkmark$ Potential for drug excipients interaction

$\checkmark$ Physiological factors that promote or inhibit the bioavailability

$\checkmark$ Biopharmaceutical factors like as regulatory status, solubilization capacity, miscibility, physical state of the excipients at room temp.

$\checkmark \quad$ Regulatory aspects of excipients

$\checkmark$ The temperature at which self-emulsification occurs

When formulating SMEDDS, drugs have to be incorporated into an appropriate mixture of excipients; therefore formulation development commonly starts with excipients selection. As there are many lipid-based substances that can be used for formulating SMEDDS, some general criteria for excipients selection were introduced in order to save time and cut costs.
During preliminary selection studies, a few excipients are identified as possibly appropriate for further research owing to their safety, drug solubility and stability in excipients, and some other characteristics. Initial selection of promising excipients is then followed by construction of phase diagrams to identify suitable mixing ratios for homogeneous formulations, being just as crucial as sufficient solubilization capacity for the drug to be incorporated. Once candidate formulations are proposed, the drug-loaded systems are subjected to in vitro dispersion and digestion tests to predict the fate of the drug in the GIT.

The in vivo behavior of the formulation can be interpreted easily by lipid formulation classification system (LFCS). With reference to the physico-chemical properties of specific drugs, the most suitable formulation can be identified through LFCS. Table 2 shows the various classes of LFCS. Most of the marketed products are Type III systems, which are diverse with a wide range of oil and water-soluble substances. Hence, this group has been further divided into Type IIIA (oils) and Type IIIB (water-soluble) based on the proportion of oils and water-soluble substances $^{5,22}$.

Table 2: The lipid formulation classification system ${ }^{5,26}$

\begin{tabular}{|c|c|c|c|c|c|c|}
\hline & Type I & Type II & Type III A & Type III B & Type IV \\
\hline \multirow{4}{*}{$\begin{array}{l}: \stackrel{0}{\Xi} \\
0 \\
0 \\
0 \\
0 \\
0\end{array}$} & Oils & $100 \%$ & $40-80 \%$ & $40-80 \%$ & $<20 \%$ & $0 \%$ \\
\hline & $\begin{array}{l}\text { Water-insoluble } \\
\text { surfactants (HLB < 12) }\end{array}$ & $0 \%$ & $20-60 \%$ & $0 \%$ & $0 \%$ & $0-20 \%$ \\
\hline & $\begin{array}{l}\text { Water-soluble } \\
\text { surfactants }(\text { HLB > 12) }\end{array}$ & $0 \%$ & +2 & $20-40 \%$ & $20-50 \%$ & $30-80 \%$ \\
\hline & $\begin{array}{l}\text { Hydrophilic co-solvents } \\
\text { (e.g. PEG, propylene } \\
\text { glycol, transcutol) }\end{array}$ & $0 \%$ & $0 \%$ & $0-40 \%$ & $20-50 \%$ & $0-50 \%$ \\
\hline \multicolumn{2}{|c|}{ Particle size } & Coarse & $100-250$ & $100-250$ & $50-100$ & $<50$ \\
\hline \multicolumn{2}{|c|}{ Characteristics } & Non-dispersing & $\begin{array}{l}\text { Emulsion } \\
\text { (SEDDS) }\end{array}$ & $\begin{array}{l}\text { SEDDS/ } \\
\text { SMEDDS } \\
\text { formed with } \\
\text { water-soluble } \\
\text { components }\end{array}$ & $\begin{array}{l}\text { SEDDS/ } \\
\text { SMEDDS } \\
\text { formed with } \\
\text { water-soluble } \\
\text { components } \\
\text { and low oil } \\
\text { content }\end{array}$ & $\begin{array}{l}\text { Disperses } \\
\text { typically to } \\
\text { form a } \\
\text { micellar } \\
\text { solution }\end{array}$ \\
\hline \multicolumn{2}{|c|}{ Digestibility } & $\begin{array}{l}\text { Requires } \\
\text { digestion }\end{array}$ & $\begin{array}{l}\text { Will be } \\
\text { digested }\end{array}$ & $\begin{array}{l}\text { Digestion may } \\
\text { not be necessary }\end{array}$ & $\begin{array}{l}\text { Digestion may } \\
\text { not be } \\
\text { necessary }\end{array}$ & $\begin{array}{l}\text { Limited } \\
\text { digestion }\end{array}$ \\
\hline \multicolumn{2}{|c|}{ Advantages } & $\begin{array}{l}\text { GRAS status; } \\
\text { simple; } \\
\text { excellent } \\
\text { capsule } \\
\text { compatibility }\end{array}$ & $\begin{array}{l}\text { Unlikely to } \\
\text { lose solvent } \\
\text { capacity on } \\
\text { dispersion }\end{array}$ & $\begin{array}{l}\text { Clear or almost } \\
\text { clear dispersion. } \\
\text { Absorption } \\
\text { without digestion }\end{array}$ & $\begin{array}{l}\text { Clear } \\
\text { dispersion. } \\
\text { Absorption } \\
\text { without } \\
\text { digestion }\end{array}$ & $\begin{array}{l}\text { Good solvent } \\
\text { capacity for } \\
\text { many drugs; } \\
\text { disperse to } \\
\text { micellar } \\
\text { solution }\end{array}$ \\
\hline \multicolumn{2}{|c|}{ Disadvantages } & $\begin{array}{l}\text { Poor solvent } \\
\text { capacity } \\
\text { (unless drug is } \\
\text { highly } \\
\text { lipophilic) }\end{array}$ & $\begin{array}{l}\text { Turbid o/w } \\
\text { dispersion }\end{array}$ & $\begin{array}{l}\text { Possible loss of } \\
\text { solvent capacity } \\
\text { on dispersion. } \\
\text { Less easily } \\
\text { digested }\end{array}$ & $\begin{array}{l}\text { Likely loss of } \\
\text { solvent } \\
\text { capacity on } \\
\text { dispersion }\end{array}$ & $\begin{array}{l}\text { Loss of } \\
\text { solvent } \\
\text { capacity on } \\
\text { dispersion; } \\
\text { may not be } \\
\text { digestible }\end{array}$ \\
\hline
\end{tabular}




\subsubsection{Oil/Lipid phase:}

The function of oil phase in self-micro emulsifying system is to solubilized the hydrophobic/lipophilic active moiety in order to improve both drug loading and bioavailability of the hydrophobic active moiety. The lipid part of the SMEDDS forms the core of the emulsion particle and is typically composed of nonpolar lipids or Class I polar lipids in Small's Lipid Classification system. $^{27}$

A wide range of lipid excipients are available from excipient suppliers. Since these lipids affect the absorption process, it is necessary to know the characteristics of various excipients ${ }^{26}$. A lipid molecule with a large hydrophobic portion compared to hydrophilic portion is desirable as it maximizes the amount of drug that can be solubilized. The most common excipients used in lipid based drug delivery are triglyceride vegetable oils. This is one class of lipid which does not present any safety issues, since they are fully digested and absorbed ${ }^{26}$. Triglycerides can be further classified as LCT, MCT and SCT. The capacity as a solvent for drugs is mainly decided by the effective concentration of ester groups ${ }^{28}$.

\section{Long chain triglycerides (LCT):}

Lipids that have fatty acid chains of 1420 carbons are categorized as $\mathrm{LCT}^{29}$. Fixed oils i.e., vegetable oils contain a mixture of glyceride esters of unsaturated long chain fatty acids. These are considered safe as they are commonly present in daily food and are easily digestible 26. Large hydrophobic portion of triglycerides is responsible for their high solvent capacity for lipophilic moieties. Though it is difficult to micro-emulsify, some marketed formulations such as Neoral (composed of olive oil which, has shown superior oral bioavailability) and Topicaine gel (composed of Jojoba oil for transdermal application) have been successfully practicing the micro-emulsification of $\mathrm{LCT}^{30}$.

Medium chain triglycerides (MCT) and related esters:

Lipids that have fatty acid chains of 612 carbons are categorized as $\mathrm{MCT}^{30}$. MCTs are the most common choice of oil for SMEDDS as they are resistant to oxidation and possess high solvent capacity compared to LCT because of their high effective concentration of ester group. MCTs produced from the distillation of coconut oil are known as glyceryl tricaprylate and comprises of saturated $\mathrm{C} 8$ and $\mathrm{C} 10$ fatty acids in the liquid state ${ }^{26}$. Labrafac CM 10, a MCT, has shown superior solubility for fenofibrate and produced wider microemulsion region at all surfactant/cosurfactant combinations than Maisine 35, which, is a $\mathrm{LCT}^{31}$.

Drug substance should possess minimum solubility of $50 \mathrm{mg} / \mathrm{ml}$ in LCTs for lymphatic absorption ${ }^{32}$. Upon digestion, products of short and medium chain triglycerides are directed towards portal vein whereas chylomicrons formed from LCTs triggers the lymphatic transport. Further, highly hydrophobic drug substances are easily soluble in vegetable oils and can easily be formulated as simple oil solutions which are readily emulsified in the gut. However, most conventional hydrophobic drug substances do not exhibit superior solubility in LCT such as vegetable oil ${ }^{33,34}$.

Moderately hydrophobic drug substances, on the other hand, cannot be formulated into simple oil solutions as their solubility is limited. In such cases, SMEDDS are promising alternative where the drug solubility in the oil will be enhanced due to micro emulsification of oil by surfactants. It is well accepted that oils with long hydrocarbon chains (high molecular volume) such as soybean oil, castor oil are difficult to micro-emulsify compared to MCT (low molecular volume) such as capmul MCM and Miglyol. However, solubilizing capacity of oil for lipophilic moiety increases with chain length (hydrophobic portion) of the oil.

Hence the selection of oil is a compromise between the solubilizing potential and ability to facilitate the formation of microemulsion ${ }^{35}$. Malcolmson et al. studied the solubility of testosterone propionate in various oils for the formulation of $\mathrm{O} / \mathrm{W}$ micro-emulsion and concluded that oils with larger molecular volume such as triglycerides show superior solubility than the corresponding micellar solution containing only surfactants without oil ${ }^{36}$. Enhancement of drug solubility in SMEDDS not only relies on the solubility of the drug in the oil but also on the surfactant(s). For instance, ethyl butyrate, small molecular volume oil, has shown higher solubility for testosterone propionate but its ME formulation has only improved the solubility slightly than the corresponding micellar solution. On the contrary, Miglyol 812 which is a larger molecular volume oil has shown improved solubilization in the ME formulation though the solubility of testosterone propionate is less in the individual components compared to ethyl butyrate ${ }^{36}$. The compositions of fatty acids found in various lipid excipients are presented in Table 3.

Grovea et al. made a direct comparison of two seocalcitol II loaded SMEDDS containing either MCT or LCT. The study was performed on monophasic systems with the same lipid/surfactant/co-surfactant ratio, which formed dispersions with the same droplet size distribution upon dilution with the aqueous phase. Cremophor ${ }^{\circledR}$ RH40 was used as surfactant in both cases, whereas the co-surfactant was chosen to resemble the lipid component in chain length. Reportedly, a larger microemulsion area was achieved in the phase diagram when MCT was used instead of LCT due to the difference in polarity between the lipids. As the more hydrophobic LCT is more difficult to emulsify, higher concentration of Cremophor® RH 40 was generally required to form microemulsions when using LCT compared to MCT. Nevertheless, no significant differences were observed in the absorption and bioavailability of seocalcitol between the two aforementioned SMEDDS upon their oral administration to male rats ${ }^{37}$. This is contrary to previous studies, where the bioavailability of danazol and halofantrine from SMEDDS containing LCT was found to be superior compared to SMEDDS containing MCT $^{38,}$, 39 . However, SMEDDS compared in these studies consisted of different amounts of lipid and surfactant, whereas Grovea et al. used quantitatively comparable systems. Considering the mentioned data, one can conclude that the extent of influence of MCT and LCT on the bioavailability of drugs is drug specific ${ }^{37}$. 
Table 3: List of oils/lipids used in formulation in SMEDDS

\begin{tabular}{|c|c|c|c|}
\hline \multicolumn{2}{|c|}{ Class } & Examples & Chracteristics \\
\hline \multirow[b]{2}{*}{ 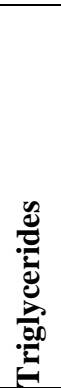 } & LCT & $\begin{array}{l}\text { Corn oil, soybean oil, olive oil, } \\
\text { peanut oil, sesame oil, } \\
\text { sunflower oil, castor oil, etc. }\end{array}$ & $\begin{array}{l}1420 \text { carbons in fatty acid chains } \\
\text { GRAS status, easily ingested, digested, and absorbed, poor self- } \\
\text { dispersing properties of LCT and generally lower loading capacity for } \\
\text { drugs with intermediate log P values. Their advantage is generally a } \\
\text { higher solubilizing capacity after dispersion and digestion of the } \\
\text { formulation }{ }^{40-42} \text {. }\end{array}$ \\
\hline & MCT & $\begin{array}{l}\text { Fractionated coconut oil, palm } \\
\text { seed oil, triglycerides of } \\
\text { caprylic/ capric acid Miglyol@ } \\
812 \text {, Captex@ } 355\end{array}$ & $\begin{array}{l}612 \text { carbons in fatty acid chains } \\
\text { MCTs exhibit a good solubilizing capacity for less lipophilic drugs } \\
\text { and good self-dispersing ability. Semisynthetic MCT with } \\
\text { hydrogenated double bonds are resistant to oxidation }{ }^{40-42} \text {. }\end{array}$ \\
\hline \multicolumn{2}{|c|}{$\begin{array}{l}\text { Mixed mono-, di- } \\
\text { and triglycerides }\end{array}$} & 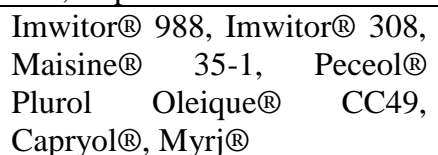 & $\begin{array}{l}\text { They possess surface active properties because of their amphiphilic } \\
\text { nature and are effective in replacing conventionally used oils owing to } \\
\text { their better self-dispersing ability and higher solubilizing capacity for } \\
\text { poorly water-soluble drugs }{ }^{41-41} \text {. }\end{array}$ \\
\hline
\end{tabular}

\subsubsection{Surfactants.}

The self-emulsifying properties require the incorporation of relatively large amounts of surfactant in the formulation in addition to the oily drug carrier vehicle. The surfactants may improve the affinity between lipids and intestinal membrane or increase the permeability of the intestinal membrane. Surfactants increase the permeability by partitioning into the cell membrane and disrupting the structural organization of the lipid bilayer leading to permeation enhancement ${ }^{43}$. Therefore, most drugs are absorbed via the passive transcellular route. They also exert their absorption enhancing effects by increasing the dissolution rate of the drug.

The selection of surfactant is also critical for the formulation of SMEDDS. In order to select a surfactant for SMEDDS formulation, due consideration must be give to HLB value and safety of surfactant. The HLB of a surfactant gives vital information on its application in formulation of SMEDDS. The surfactant/emulsifier involved in the formulation of SMEDDS should have a relatively high HLB and hydrophilicity to enable rapid and facile dispersion in the aqueous GI fluid as a very fine oil-in-water emulsion, and hence good selfemulsifying performance can be achieved ${ }^{44}$. The use of surfactant blends to achieve the HLB required for emulsification has often been proven to provide superior self-emulsifying properties relative to the use of a single surfactant possessing the desired HLB ${ }^{45}$.

Various vegetable oil derivatives like Acrosyl (castor oil derivative) are still being found to give optimum selfemulsification $^{46}$. Nonionic surfactants are normally preferred over their ionic counterparts due to lower toxicity and greater emulsion stability over a wider range of $\mathrm{pH}$ and ionic strength. On the other hand, a possible disadvantage is their influence on the permeability of intestinal lumen with a reversible effect ${ }^{43}$, further facilitating absorption of the co-administered drug. Hydrophobic surfactants can penetrate membranes causing changes in membrane fluidity and permeability.

Safety is a major determining factor in choosing a surfactant. Emulsifiers of natural origin (e.g., lecithin, Akoline medium chain monoglycerides (MCM), and Peceol) are normally preferred since they are considered to be safer than the synthetic surfactants. However, these excipients have limited self-emulsification efficiency ${ }^{41}$.

Generally single alkyl chains are more penetrative, so bulky surfactants such as polysorbates and triglyceride ethoxylates are found to be less toxic. Usually the surfactant concentration ranges between 30 and $60 \%$ of the total formulation in order to form stable SMEDDS ${ }^{47}$. It is very important to determine the surfactant concentration properly as large amounts of surfactants may cause GI irritation. However, the extremely small lipid droplet size produced by SMEDDS formulations promotes rapid stomach emptying and wide dispersion throughout the GIT, minimizing exposure to high local surfactant concentrations and thus reducing the irritation potential.

\subsubsection{Co-surfactants/Co-solvents}

Usually, the formulation of a successful SMEDDS requires high concentrations of surfactant (up to 50\%) and addition of co-surfactants aids in selfemulsification. Generally co-surfactant of HLB value 10-14 is used with surfactant to decrease the oil-water interfacial tension, fluidize the hydrocarbon region of interfacial film, increase the drug loading to SMEDDS and allows the spontaneous formation of microemulsion. $^{48}$ Hence, surfactants (hydrophilic or lipophilic) and/or amphiphilic solubilizers are used for this purpose. The addition of the co-emulsifiers or solubilizers in SMEDDS may result in an expanding self-micro-emulsification region in the phase diagrams.

Organic solvents such as, ethanol, PEG and PG are suitable co-solvents for oral delivery, and they enable the solubilization of large quantities of either the hydrophilic surfactant or the drug in the lipid base. The lipid mixture with higher surfactant and co-surfactant: oil ratios leading to the formation of $\operatorname{SMEDDS}^{49}$. Alcohol and other volatile co-solvents have evaporated into the shell of soft or hard gelatin capsules, results in precipitation of drug.

\subsection{Mechanism of self-emulsification}

Self-emulsification occurs when the entropy change that favors dispersion is greater than the energy required to increase the surface area of the dispersion. The free energy of the conventional emulsion is a direct function 
of the energy required to create a new surface between the water and oil phases and can be described by the equation:

$$
\Delta \mathrm{G}=\sum \mathrm{N}_{\mathrm{i}} \pi \mathrm{r}_{\mathrm{i}}^{2} \mathrm{~S}
$$

Where $\Delta \mathrm{G}$ is the free energy associated with the process (ignoring the free energy of mixing), $\mathrm{N}$ is the number of droplets of radius $r$ and $S$ represents the interfacial energy. The two phases of emulsion tend to separate with time to reduce the interfacial area and, subsequently, the emulsion is stabilized by emulsifying agents, which form a monolayer of emulsion droplets, and hence reduces the interfacial energy, as well as providing a barrier to prevent coalescence Reiss ${ }^{50,51}$.

The above equation shows that spontaneous formation of interface between oil and aqueous phase is thermodynamically stable Reiss ${ }^{50}$ explained the spontaneous formation of emulsion, i.e., selfemulsification, in terms of the free energy required to form the emulsion which is either very low and positive, or negative.

Pouton $^{52}$ has proposed a relationship between the emulsification properties of the surfactant and phase inversion behavior of the system. For example, the temperature of the oil in water system, stabilized by using non-ionic surfactant(s) is increased; the cloud point of the surfactant would be attained followed by phase inversion. The surfactant is highly mobile at the phase inversion temperature; hence, the o/w interfacial energy is minimized, results to a reduction in energy needed for emulsification.

\subsection{Characterizations of SMEDDS}

The various ways to characterize SMEDDS are compiled below;

\section{Visual assessment}

The primary means of self-emulsification assessment is visual evaluation. This may provide key information about the self emulsifying and micro-emulsifying property of the mixture and about the resulting dispersion.

\section{Equilibrium phase diagram}

Comparison of different surfactants and their synergy with co-solvent is enabled using equilibrium phase diagram. The boundaries of one phase region can easily be assessed visually. The phase behavior of a threecomponent system can be represented by a ternary phase diagram. Phase diagram helps in determining the optimum concentrations of different excipients necessary to obtain homogenous pre-concentrates, selfemulsifying ability and drug loading. Each corner of phase diagram represents $100 \%$ of particular components and when more than three components are used, closely related one are grouped together as one component and treated as such in the diagram. ${ }^{52}$

\section{Turbidity measurement}

This determines the efficiency of self-emulsification by establishing whether the dispersion reaches equilibrium rapidly and in a reproducible time ${ }^{53}$. These measurements are carried out using turbidity meters
(Hach turbidity meter and the Orbeco-Helle turbidity meter). ${ }^{54}$.

\section{Droplet size}

Microscopic techniques, Photon correlation spectroscopy or a Coulter Nanosizer are generally used to determine the droplet size of emulsion. Droplet size is an important factor in self-emulsification performance because it determines the rate and extent of drug release, as well as the stability of the microemulsion ${ }^{55}$.

\section{Electron microscopic studies}

Surface characteristics of micro-emulsion are studied using Freeze-fracture electron microscopy ${ }^{56}$.

\section{Zeta potential measurement}

It is used to identify the charge of the droplets.

\section{Determination of emulsification time}

This process is used for estimation of the time taken for emulsification. In this efficiency of emulsification of various compositions of the surfactants and lipids is quantified using a rotating paddle to promote emulsification in a crude nephelometer ${ }^{34}$.

\section{Particle size distribution}

Dynamic light scattering techniques is used for measurement of particle size distribution of the microemulsion. This utilizes the fluctuation in scattered light intensity to measure the velocity of the Brownian diffusion and consequently the dispersed droplets. Particle size distributions can be further verified by cryogenic transmission electron microscopy (cryoTEM). Cryo-TEM offers the advantage of visualizing the particle sizes and shapes. ${ }^{57}$

\section{Conductivity measurements}

Conductivity measurements are able to determine the point of aqueous phase addition where the system changes from having oil continuous to a water continuous phase. It also helps in monitoring of percolation or phase inversion phenomena. ${ }^{58}$

\section{BIOPHARMACEUTICAL ASPECTS OF SMEDDS}

These systems increase absorption from the gastrointestinal tract by accelerating the dissolution process, facilitating the formation of solubilized phases by reduction of particle size to the molecular level, yielding a solid state solution within the carrier ${ }^{59}$, changing drug uptake, efflux and disposition by altering enterocyte based transport ${ }^{60}$, and enhancing drug transport to the systemic circulation via intestinal lymphatic system ${ }^{61}$

\subsection{Effect of Lipids}

The effect of lipids on the bioavailability of orally administered drugs is highly complex due to numerous mechanisms by which the lipids can alter the biopharmaceutical characteristics of the drug. Factors such as the acid chain length of triglyceride, the saturation degree and the volume of lipid administered may affect the drug absorption profile and its blood/lymph distribution. 


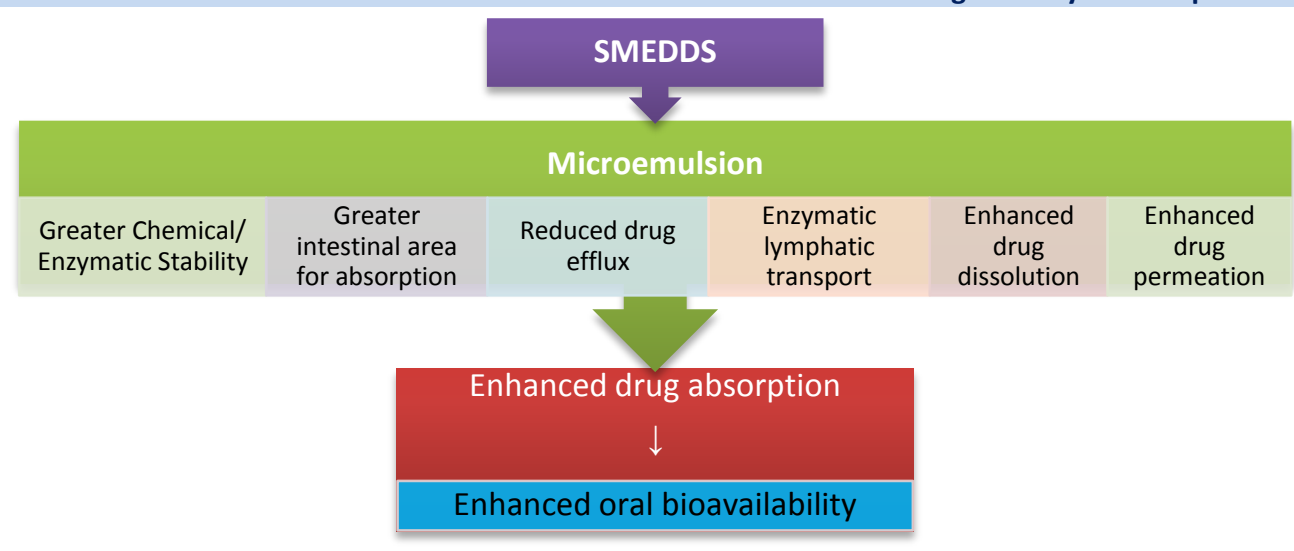

Figure 2: Main factors that potentially affect the bioavailability of drugs formulated in SMEDDS

\subsubsection{Effect on rate of gastric emptying}

Increase in gastric residence time shows the delivery of the drug of it site of action. In particular, it is the lipid component of the food that plays a vital role in the absorption of lipophilic drugs. Lipids in the GI tract provoke delay in gastric emptying, i.e. gastric transit time is increased leading to enhanced oral bioavailability co-administered lipophilic drug ${ }^{67}$.

This can be explained by the ability of a high fat meal to stimulate biliary and pancreatic secretions, to decrease metabolism and efflux activity, to increase intestinal wall permeability, and to a prolongation of GIT residence time and transport via lymphatic system ${ }^{68}$. Triglycerides and long chain fatty acids play a major role in prolonging the GIT residence time.

\subsubsection{Effect on Digestion and solubilization of drug}

The balance between a drug's solubility in the aqueous environment of the gastrointestinal lumen and its permeation across the lipophilic membrane of enterocytes determines its rate and extent of absorption $^{60}$.

Following ingestion, of SMEDDS, gastric lipase initiates the digestion of exogenous dietary $\mathrm{TG}$ and formulation TG. Simultaneously, the mechanical mixing (propulsion, grinding and retropulsion) of the stomach facilitates formation of a crude emulsion (comprised of aqueous gastric fluid and lipid digestion products).
Later in the small intestine, pancreatic lipase together with its cofactor co-lipase 203 completes the breakdown of TG to diglyceride, monoglyceride and fatty acid. Pancreatic lipase acts primarily at the sn-1 and sn-3 positions of TG to produce 2-monoglyceride and free fatty acid $^{69,70}$. The chemical digestion of formulation- or biliary-derived phospholipid (PL) also occurs in the small intestine in which pancreatic phospholipase A2 hydrolyses a single fatty-acid molecule from the sn-2 position of PL to yield lysophosphatidylcholine and fatty $\operatorname{acid}^{71}$.

The presence of exogenous lipids in the small intestine also stimulates secretion of endogenous biliary lipids, including bile salt (BS), PL and cholesterol from the gall bladder. Previously formed monoglycerides, fatty acids, and lysophospholipid (products of lipid digestion) are subsequently in corporate into a series of colloidal structures, including micelles and unilamellar and multilamellar vesicles in the presence of bile salts. The solubilization and absorptive capacity of the small intestine for lipid digestion products and drugs (D) is significantly enhanced due to these formed lipid metabolites. In Fig. 3, the oil droplet in the intestine is represented in different colors to indicate undigested TG in the core (orange) and digested products such as fatty acid (blue) and monoglyceride (green) on the surface of the droplet.

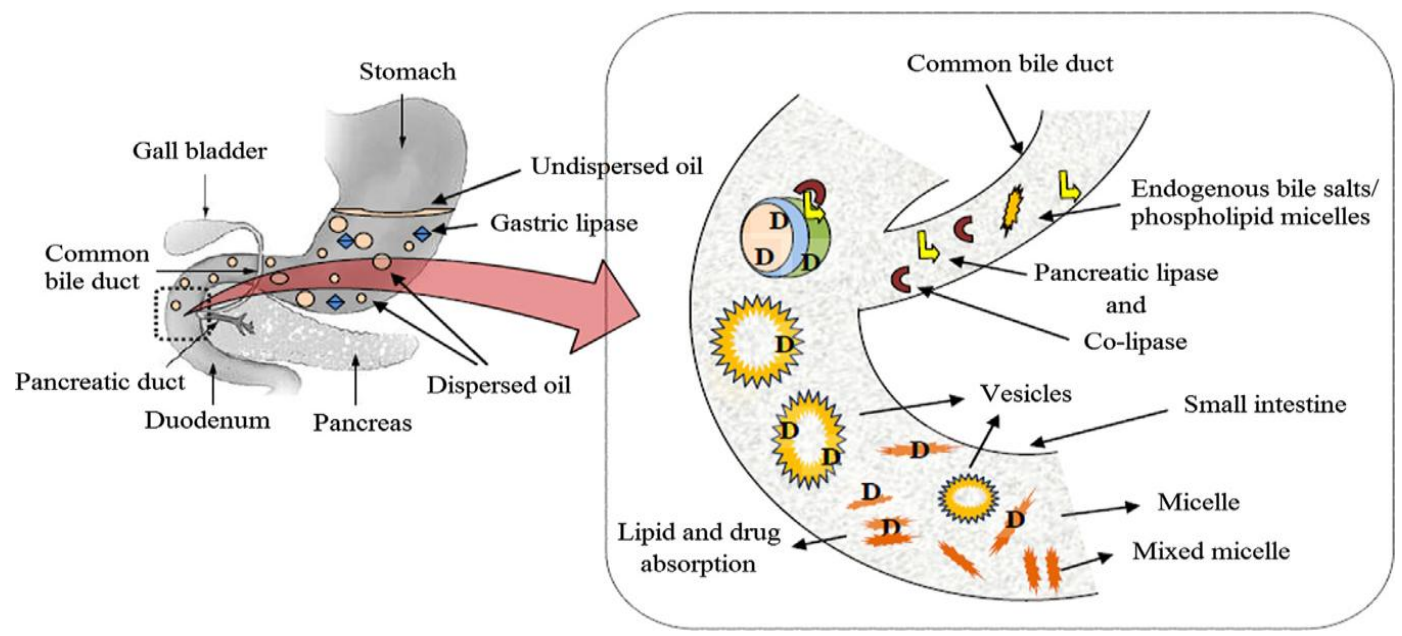

Figure 3: Lipid digestion and drug solubilization process in the small intestine ${ }^{72}$ 
Following their penetration through the aqueous layer and mucin, mixed micelles and micro-emulsions are absorbed either by pinocytosis, diffusion or endocytosis. The drug compound then reaches the systemic circulation via the portal vein or lymphatic system

\subsubsection{Promotion of intestinal lymphatic transport}

For highly lipophilic drugs, lipids may enhance the extent of lymphatic transport and increase bioavailability directly or indirectly via a reduction in first-pass metabolism ${ }^{\mathbf{6 2 - 6 4}}$. Lipids increase the TG-rich lipoproteins which react with drug molecules. Lipoproteins-drug complex enhances intestinal lymphatic transport and leads to changes in drug disposition and finally changes the kinetics of the pharmacological actions of poorly soluble drugs ${ }^{73}$.

The effect of structured triglycerides with varying intra molecular structures and chain lengths incorporated into a SMEDDS on the intestinal lymphatic transport and absorption of halofantrine into the blood was investigated ${ }^{74}$. The SMEDDS formulation included $29 \% \mathrm{w} / \mathrm{w}$ structured triglyceride designated as LLL, LML, or MLM (L: long chain fatty acid, C18; M: medium chain fatty acid, C8-10). The MLM and LML micro-emulsions had a similar droplet size of $50 \mathrm{~nm}$. The lymphatic transport of halofantrine, expressed as the cumulative percentage of the administered dose, after $12 \mathrm{~h}$ (mean \% dose \pm S.E.) was $27.4 \pm 1.3$ after administration in the LML and $17.9 \pm 1.3$ in the MLM. The results indicated that the structural formation of the triglyceride initiated a lymphatic transport at a high level. It was therefore hypothesized that medium chain fatty acid enhanced the absorption into the systemic blood circulation whereas long chain fatty acid enhanced the lymphatic transport. Thus, the absorption profile of a drug formulated into a SMEDDS could be manipulated by varying the medium and long chain triglyceride content in the formulation in order to improve the oral bioavailability of highly lipophilic drugs.

\subsubsection{Effect on intestinal permeability}

Oil component alters the solubility of the drug in SMEDDS by penetrating into the hydrophobic portion of the surfactant monolayer. Extent of oil penetration varies and depends on the molecular volume, polarity, size and shape of the oil molecule. Overall drug solubility in SMEDDS is always higher than the solubility of drug in individual excipients that combine to form SMEDDS. However, such higher solubility considerably depends on the solubility of drug in oil phase, interfacial locus of the drug and drug-surfactant interactions at the interface ${ }^{75}$.

In light scattering experiments, it was observed that oils with small molecular volume act like co-surfactants and penetrate into the surfactant monolayer. This forms thinner polyoxyethylene chains near the hydrophobic core of the micelle disrupting the main locus of the drug solubilization due to which, a higher solubility of drug is not observed.

Large molecular volume oils, however, forms a distinct core and do not penetrate effectively into the surfactant monolayer. The locus of drug solubilization was found to be effected by the microstructure and solubility of the drug in the excipients. The locus of drug solubilization was found to be at the interface of micelle for phytosterols whereas the same for cholesterol was found to be between the hydrophobic head groups of surfactant molecules. This is attributed to altered side chain flexibility of phytosterol due to the additional substitution of alkyl side chain compared to cholesterol $^{76}$.

In addition to molecular volume and polarity of the oil, drug solubility in oil is affected by physicochemical properties of drug molecule itself. Consideration of BCS classification and Lipinski's rule of 5 for the selection of drug is only useful during initial screening stages. As per BCS classification, some of the acidic drugs are listed in Class II despite having good absorption and disposition as they do not satisfy the requirement of higher solubility at low $\mathrm{pH}$ values. Lipinski's rule of 5, on the other hand, holds good only when the drug is not a substrate for the active transporter ${ }^{77}$. This suggests that aqueous solubility and $\log \mathrm{P}$ alone are not sufficient to predict the solubility of drug in the oil. This further indicates that the solubility of any two drugs with similar $\log \mathrm{P}$ would not be the same due to their different physicochemical properties.

\subsubsection{Reduced metabolism and efflux activity}

In some cases, as shown recently, excipients incorporated in SMEDDS can inhibit both pre-systemic drug metabolism and intestinal efflux mediated by P-gp resulting in an increased oral absorption of cytotoxic drugs ${ }^{65-66}$. It is clear that certain lipids and surfactants may attenuate the activity of intestinal efflux transporters, as indicated by the p-glycoprotein efflux pump, and may also reduce the extent of enterocyte-based metabolism ${ }^{65,78}$. Therefore uptake of lipophilic drugs formulated as SMEDDS from the GI tract can enhanced due to decrease in the P-gp drug efflux $^{79}$. In addition to a multidrug efflux pump, phase I metabolism by the intestinal Cytochrome P450s is now becoming recognized as a significant factor in oral drug bioavailability. ${ }^{80}$

\subsection{Effect of surfactants}

\subsubsection{Effects on permeability}

Surfactants increase the permeability by interfering with the lipid bilayer of the single layer of the epithelial cell membrane ${ }^{81}$, which with the unstirred aqueous layer, forms the rate-limiting barrier to drug absorption/diffusion ${ }^{62}$. Therefore, most drugs are absorbed via the passive transcellular route. Surfactants partition into the cell membrane and disrupt the structural organization of the lipid bilayer leading to permeation enhancement. They also exert their absorption enhancing effects by increasing the dissolution rate of the $\operatorname{drug}^{83}$. They also exert their absorption enhancing effects by increasing the dissolution rate of the $\operatorname{drug}^{84}$.

\subsubsection{Effect on Droplets size}

The lipid mixtures with higher surfactant and cosurfactant/oil ratios lead to the formation of SMEDDS 
85 . The surfactant concentration required to form a stable SMEDDS ranges from 30 to $60 \%(\mathrm{~m} / \mathrm{m})^{86}$. The lowest possible surfactant concentration should be used in order to prevent gastric irritation. The extremely small droplet size produced in the case of SMEDDS promotes rapid gastric emptying and low local concentration of the surfactant, thereby reducing gastric irritation.

There is a relationship between the droplet size and the concentration of the surfactant being used. Surfactant concentration has been shown to have varying effects on the droplet size of emulsion. Increase in surfactant concentration causes a decrease in droplet size associated with stabilization of surfactant molecules at the oil-water interface, although the reverse is possible due to enhanced water penetration into oil droplets leading to their breakdown ${ }^{43}$.

In some cases, increasing the surfactant concentration could lead to droplets with smaller mean droplet size such as in the case of a mixture of saturated C8-C10 polyglycolized glycerides (Labrafac CM-10). This could be explained by the stabilization of the oil droplets as a result of the localization of the surfactant molecules at the oil-water interface ${ }^{87}$. On the other hand, in some cases the mean droplet size may increase with increasing surfactant concentrations ${ }^{88}$. This phenomenon could be attributed to the interfacial disruption elicited by enhanced water penetration into the oil droplets mediated by the increased surfactant concentration and leading to ejection of oil droplets into the aqueous phase.

The role of surfactants in SMEDDS is to reduce the interfacial tension and adjust the spontaneous curvature of the interface so as to enable the dispersion process and provide a flexible film that can easily cover the lipid

\section{REFERENCES}

1. Pande SV, Biyani KR, Microencapsulation by solvent evaporation method of BCS Class 4 drugs for bioavailability enhancement, Journal of Drug Delivery and Therapeutics. 2016; 6(5):18-30.

2. Mundhe AV, Fuloria NK, Biyani KR, Cocrystalization: an alternative approach for solid modification, Journal of Drug Delivery and Therapeutics. 2013; 3(4):166-172.

3. Shelke PV, Dumbare AS, Gadhave MV, Jadhav SL, Sonawane AA, Gaikwad DD, Formulation and evaluation of rapidly dis integrating film of amlodipine bes ylate, Journal of Drug Delivery \& Therapeutics; 2012; 2(2):72-75.

4. Abdalla A, Sandra K, Mader K. A new self-emulsifying drug delivery system (SEDDS) for poorly soluble drugs: characterization, dissolution, in vitro digestion and incorporation into solid pellets. Eur J Pharm Sci 2008; 35(5):457-464.

5. Pouton CW. Formulation of poorly water-soluble drugs for oral administration: physicochemical and physiological issues and the lipid formulation classification system. Eur $\mathrm{J}$ Pharm Sci 2006; 29(3e4):278e287.

6. Chandel P, Kumari R, Kapoor A, Liquisolid technique: an approach for enhancement of solubility, Journal of drug delivery and therapeutics, 2013; 3(4):131-137

7. Miryala V, Kurakula M, Self-nano emulsifying drug delivery systems (SNEDDS) for oral delivery of atorvastatinformulation and bioavailability studies, Journal of Drug Delivery \& Therapeutics, 2013; 3(3):131-142.

8. Singh J, Walia M, Harikumar SL, Solubility enhancement by solid dispersion method: a review, Journal of drug delivery and Therapeutics, 2013; 3(5):148-155. core of the emulsion droplets and lead to the spontaneous formation of a nano- or micro-emulsion. Basically, the increase of surfactant activity at the water-oil interface would result in a decrease of interfacial tension. Moreover, the addition of a second surfactant to the system would usually cause a further decrease in interfacial tension down to a very small, even transient negative value, at which the interface would expand to form fine dispersed droplets.

\section{CONCLUSION}

Lipid-based drug delivery systems, especially SMEDDS, are a promising approach for improving the bioavailability of poorly soluble drug. Bioavailability enhancement have been attributed to a number of factors, including delivery of the drug in solution to the gastrointestinal tract, increased bile secretion, easier partition of the drug into the mixed micelles that are believed to facilitate drug absorption, stimulation of gastric lymphatic transport and increased intestinal permeability.

The effect of lipids on the bioavailability of orally administered drugs is highly complex due to numerous mechanisms by which the lipids can alter the biopharmaceutical characteristics of the drug. Better understanding of the role of individual lipids, surfactants and co-surfactants in the formation of SMEDDS, with regard to the dispersion process, the structure of the formed emulsion particle and drug solubilisation is very important in successful designing of these formulations. Therefore this review focused on the physic-chemical and biopharmaceutical aspects of the SMEDDS which may be helpful for the advancement of this technology to obtain a safer, more stable and efficacious SMEDDS formulations.

9. Dalvi PB, Gerange AB, Ingale PR, Solid dispersion: strategy to enhance solubility, Journal of Drug Delivery and Therapeutics. 2015; 5(2):20-28.

10. Aungst BJ. Novel formulation strategies for improving oral bioavailability of drugs with poor membrane permeation or presystemic metabolism. J Pharm Sci 1993; 82:979-987.

11. Shah DP, Patel B, Shah C, Nanosuspension technology: A innovative slant for drug delivery system and permeability enhancer for poorly water soluble drugs, Journal of Drug Delivery and Therapeutics, 2015; 5(1):10-23.

12. Venkatesh, G. et al. In vitro and in vivo evaluation of selfmicroemulsifying drug delivery system of buparvaquone. Drug Dev. Ind. Pharm. 2010; 36:735-745.

13. Gursoy, R.N. and Benita, S. Self-emulsifying drug delivery systems (SEDDS) for improved oral delivery of lipophilic drugs. Biomed. Pharmacother. 2004; 58:173-182.

14. Singh, B. et al. Self-emulsifying drug delivery systems (SEDDS): formulation development, characterization, and applications. Crit. Rev. Ther. Drug Carrier Syst. 2009; 26:427-521.

15. Charman WN, Stella VJ. Transport of lipophilic molecules by the intestinal lymphatic system. Adv Drug Del Rev. 1991; 7:114.

16. Porter CJ, Trevaskis NL, Charman WN. Lipids and lipidbased formulations: optimizing the oral delivery of lipophilic drugs. Nat Rev Drug Discov. 2007; 6:231-248.

17. Sanghai B, Aggarwal G, HariKumar SL, Solid self microemulsifying drug deliviry system: a review, Journal of Drug Delivery and Therapeutics. 2013; 3 (3):168-174 
18. Dhakar RC, Maurya SD, Saluja V. From formulation variables to drug entrapment efficiency of microspheres: A technical review, Journal of Drug Delivery \& Therapeutics. 2012; 2(6): 128-133.

19. de Smidt PC et al. Intestinal absorption of penclomedine from lipid vehicles in the conscious rat: contribution of emulsification versus digestibility. Int J Pharm 2004; 270 : 109-118.

20. Shah NH, Carvajal MT, Patel CI, Infeld MH, Malick AW Selfemulsifying drug delivery systems (SEDDS) with polyglycolysed glycerides for improving in vitro dissolution and oral absorption of lipophilic drugs. Int J Pharm . 1994; 106:15-23.

21. Charman SA, Charman WN, Rogge MC, Wilson TD, Dutko FJ , Pouton CW . Self-emulsifying drug delivery systems: formulation andbiopharmaceutic evaluation of an investigational lipophilic compound. Pharm Res. 1992; 9 : 87 -93 .

22. Pouton CW. Lipid formulations for oral administration of drugs: non-emulsifying, self-emulsifying and 'self microemulsifying' drug delivery systems. Eur J Pharm Sci . 2000; 11:S93 - S98

23. Kommuru TR, Gurley B, Khan MA, Reddy IK. Selfemulsifying drug delivery systems (SEDDS) of coenzyme Q10: formulation development and bioavailability assessment. Int J Pharm. 2001; 212:233 - 246

24. O'Driscoll, C.M. and Griffin, B.T. Biopharmaceutical challenges associated with drugs with low aqueous solubility the potential impact of lipid-based formulations. Adv. Drug Deliv. Rev. 2008; 60:617-624

25. Kohli K, Chopra S, Dhar D, Arora S, Khar RK, Selfemulsifying drug delivery systems: an approach to enhance oral bioavailability, Drug Discovery Today, 2010; 15(21/22):958-965.

26. Pouton CW, J. H. C. Porter, Formulation of lipid-based delivery systems for oral administration: Materials, methods and strategies, Adv. Drug Deliver. Rev. 2008; 60:625-637; DOI: $10.1016 /$ j.addr.2007.10.010.

27. Small DM. A classification of biologic lipids based upon their interaction in aqueous systems. J Am Oil Chem Soc 1968; 45: 108-119.

28. Cao Y, Marra M, Anderson BD. Predictive relationships for the effects of triglyceride ester concentration and water uptake ons olubility and partitioning of small molecules into lipid vehicles. J Pharm Sci 2004; 93:2768-79.

29. Prajapati NH, Patel DP, Patel NG, Dalrymple DM, Serajuddin AT. Effect of difference in fatty acid chain lengths of medium chain lipids on lipid/surfactant/water phase diagrams and drug Solubility. J Excipients Food Chem. 2011; 2:73.

30. El Laithy HM, ElShaboury KM. The development of cutinalipogels and gel micromeulsion for topical administration of fluconazole. AAPS PharmSciTech. 2002; 3:E35. [PMCID: PMC2751344] [PubMed: 12916929]

31. Patel AR, Vavia PR. Preparation and in vivo evaluation of SMEDDS (Self Microemulsifying Drug Delivery System) containing fenofibrate. AAPS J. 2007; 9:E344-52. [PMCID: PMC2751486] [PubMed: 18170981]

32. Yanez JA, Wang SW, Knemeyer IW, Wirth MA, Alton KB. Intestinal lymphatic transport for drug delivery. Adv Drug Deliv Rev. 2011; 63:923-42. [PubMed: 21689702]

33. Stegemann S, Leveiller F, Franchi D, de Jong H, Lindén H. When poor solubility becomes an issue: From early stage to proof of concept. Eur J Pharm Sci. 2007; 31:249-61. [PubMed: 17616376]

34. Pouton CW. Formulation of selfemulsifying drug delivery systems. Adv Drug Deliv Rev. 1997; 25:47- 58.

35. Patravale VB, Date AA. Microemulsions: Pharmaceutical Applications. In: Stubenrauch C, editor. Microemulsions: Background, New Concepts, Applications, Perspectives. United Kingdom: Blackwell Publishing Ltd; 2009. pp. 25993.

36. Malcolmson C, Satra C, Kantaria S, Sidhu A, Lawrence MJ. Effect of oil on the level of solubilization of testosterone propionate into nonionic oilinwater microemulsions. J Pharm Sci. 1998; 87:109-16. [PubMed: 9452978].
37. Grovea M, Mullertzb A, Bioavailability of seocalcitol II: development and characterization of self-microemulsifying drug delivery systems (SMEDDS) for oral administration containing medium and long chain triglycerides, Eur. J. Pharm. Sci. 2006; 28:233-234; DOI: 10.1016/j.ejps.2006.02.005.

38. Khoo SM, Humberstone AJ, Porter CJH, Edwards GA, Charman WN, Formulation design and bioavailability assessment of lipidic self-emulsifying formulations of halofantrine, Int. J. Pharm. 1998; 167:155-164; DOI: 10.1016/S03785173(98)00054-4.

39. Porter CJ, Kaukonen AM, Boyd BJ, Susceptibility to lipasemediated digestion reduces the oral bioavailability of danazol after administration as a medium-chain lipid-based microemulsion formulation, Pharm. Res. 2004; 8:1405-1412; DOI: 10.1023/B:PHAM.0000036914.22132.cc.

40. Stuchlik M, Wák S, Lipid-based vehicle for oral drug delivery, Biomed. Pap. 2001; 145:17-26.

41. Constantinides PP, Lipid for improving drug dissolution and oral absorption: physical and biopharmaceutical aspects, Pharm. Res. 1995; 12:1561-1572; microemulsions DOI: 10.1023/A: 1016268311867.

42. Constantinides PP, Scalart JP, Formulation and physical characterization of water-in-oil microemulsions containing long- versus medium-chain glycerides, Int. J. Pharm. 1997; 158:57-68; DOI: 10.1016/S0378-5173(97)00248-2.

43. Swenson ES, Milisen WB, Curatolo W, "Intestinal permeability enhancement: efficacy, acute local toxicity, and reversibility," Pharmaceutical Research, vol. 1994; 11(8):1132- 1142.

44. Hauss DJ, "Oral lipid based Formulations-Enhancing the Bioavailablity of Poorly water soluble drugs," in Drugs and Pharmaceutical Sciences, 2007; 170:1-339, Informa healthcare, NC, USA.

45. Wang L, Dong J, Chen J, Eastoe J, Li X, "Design and optimization of a new self-nanoemulsifying drug delivery system," Journal of Colloid and Interface Science, 2009; 330(2):443-448.

46. Shah SR, Parikh RH, Chavda JR et al., "Self-nanoemulsifying drug delivery system of glimepiride: design, development, and optimization," PDA Journal of Pharmaceutical Science and Technology, 2013; 67(3):201-213.

47. Pouton CW, "Self-emulsifying drug delivery systems: assessment of the efficiency of emulsification," International Journal of Pharmaceutics, vol. 1985; 27(2-3):335-348.

48. Ozawa K, Olsson U, Kunieda H, Oil-induced structural change in nonionic microemulsions. J. Dispersion Sci. Technol., 1986; 22:119-124. CrossRef | Direct Link

49. Meinzer, A. et al. Microemulsion: a suitable galenical approach for the absorption enhancement of low soluble compounds? BT Gattefosse 1995; 88:21-26

50. Reiss, H. Entropy induced dispersion of bulk liquids. J. Colloid Interface Sci. 1975; 53:61-70

51. Craig D.Q. et al. An investigation into the mechanism of self emulsification using particle size analysis and low frequency dielectric spectroscopy. Int. J. Pharm. 1995; 114:103-110

52. Pouton, C.W. et al. Self-emulsifying systems for oral delivery of drugs. International Symposium on Control Release Bioactive Materials pp. 1987; 113-114.

53. Gursoy, N. et al. Excipient effects on in vitro cytotoxicity of a novel paclitaxel self-emulsifying drug delivery system. J. Pharm. Sci. 2003; 92:2411-2418.

54. Palamakula A. Khan M.A. Evaluation of cytotoxicity of oils used in coenzyme Q10 self-emulsifying drug delivery systems (SEDDS). Int. J. Pharm. 2004; 273:63-73.

55. Goddeeris C. et al. Light scattering measurements on microemulsions: estimation of droplet sizes. Int. J. Pharm. 2006; 312:187-195.

56. Vyas S.P., Khar R.K. Submicron emulsion. In Targeted and Controlled Drug Delivery Novel Carriers Systems. CBS Publishers and Distributors, 2002; pp. 291-294.

57. Fatouros DG et al. Morphological observations on a lipidbased drug delivery system during in vitro digestion. Eur $J$ Pharm Sci 2007; 31:85-94. 
58. Greiner RW, Evans DF. Spontaneous formation of a watercontinuous emulsion from water-in-oil microemulsion. Langmuir 1990; 6: 1793-1796.

59. Humberstone AJ, Charman WN. Lipid-based vehicles for the oral delivery of poorly water-soluble drugs. Adv Drug Deliv Rev 1997; 25:103-28.

60. Pramod K, Peeyush K, Rajeev K, Nitish K, Rakesh K. An overview of lipid based formulation for oral drug delivery. Drug Inv Today 2010; 2:390-5.

61. Driscoll CMO. Lipid based formulations for intestinal lymphatic delivery. Eur J Pharm Sci 2002; 15:405-15.

62. Hauss DJ, Fogal SE, Ficorilli JV, Price CA, Roy T, Jayaraj AA, Keirns JJ, Lipid-based delivery systems for improving the bioavailability and lymphatic transport of a poorly watersoluble LTB4 inhibitor, J. Pharm. Sci. 1998; 87:164-169.

63. Charman WN, Lipid vehicle and formulation effects on intestinal lymphatic drug transport, in: W.N. Chasmar, V.J. Stella (Eds.), Lymphatic Transport of Drugs, CRC Press, Boca Raton, FL, 1992, pp. 113-179.

64. Hauss DJ, Mehta SC, Radebaugh GW, Targeted lymphatic transport and modi®ed systemic distribution of CI-976, a lipophilic lipidregulator drug, via a formulation approach, Int. J. Pharm. 1994; 108:85-93.

65. Dintaman JM, Silverman JA. Inhibition of P-glycoprotein by $\mathrm{D} \square$ alpha-tocopheryl polyethylene glycol 1000 succinate (TPGS). Pharm Res 1999; 16:1550-6.

66. Chervinsky DS, Brecher BL, Hoelcle MJ. Cremophor EL enhances taxol efficacy in a multidrug resistant $\mathrm{C} 1300$ neuroblastoma cell line. Anticancer Res 1993; 13:93-6.

67. Hunt JN, Knox MT. A relation between the chain length of fatty acids and the slowing of gastric emptying. J Physiol 1968; 194:327-36.

68. Wagnera D, Langguth HS, Hanafy A, Koggela A, Langguth P. Intestinal drug efflux: formulation and food effects. Adv Drug Del Rev 2001; 50(Suppl. 1):S13-31.

69. Erlanson-Albertsson C. Pancreatic colipase. Structural and physiological aspects. Biochim. Biophys. Acta 1992; 1125:17.

70. Borgstrom, B. On the mechanism of pancreatic lipolysis of glycerides. Biochim. Biophys. Acta 1954; 13:491-504.

71. van den Bosch H., Postema N. M., de Haas G. H. \& van Deenen L. L. On the positional specificity of phospholipase A from pancreas. Biochim. Biophys. Acta 1965; 98:657-659.

72. Kalepun S, Manthina M, Padavala V, Oral lipidbaseddrugdeliverysystems - an overview, Acta Pharmaceutica Sinica B, 2013; 3(6):361-372.

73. Gershkovich P, Hoffman A. Effect of a high-fat meal on absorption and disposition of lipophilic compounds: the importance of degree of association with triglyceride-rich lipoproteins. Eur J Pharm Sci 2007; 32:24-32.

\section{Abbreviations:}

SMEDDS: self-micro emulsified drug delivery system

SEDDS: Self-emulsifying drug delivery systems

LFCS: Lipid formulation classification system

MCM: Medium chain monoglycerides

GI: gastrointestinal

HPMC: hydroxypropyl methylcellulose

P-gp: P-glycoprotein
74. Holm R, Porter CJH, Edwards GA, Müllertz A, Kristensen HG, Charman WN. Examination of oral absorption and lymphatic transport of halofantrine in a triple-cannulated canine model after administration in self-microemulsifying drug delivery systems (SMEDDS) containing structured triglycerides. Eur J Pharm Sci 2003; 20:91-7.

75. Narang AS, Delmarre D, Gao D. Stable drug encapsulation in micelles and microemulsions. Int J Pharm. 2007; 345:9-25. [PubMed: 17945446]

76. Rane SS, Anderson BD. What determines drug solubility in lipid vehicles: Is it predictable? Adv Drug Deliv Rev. 2008; 60:638-56. [PubMed: 18089295]

77. O'Driscoll CM, Griffin BT. Biopharmaceutical challenges associated with drugs with low aqueous solubilityThe potential impact of lipidbased formulations. Adv Drug Deliv Rev. 2008; 60:617-24. [PubMed: 18155800]

78. Benet L. The drug efflux-metabolism alliance: biochemical aspects. Adv Drug Deliv Rev 2001; 50: S3-S11.

79. Yu L, Bridgers A, Polli J, Vickers A, Long S, Roy A, Winnike $\mathrm{R}$, Coffin M. Vitamin E-TPGS increases absorption flux of an HIV protease inhibitor by enhancing its solubility and permeability. Pharm Res 1999; 16:1812-7.

80. Woo JS, Lee CH, Shim CK, Hwang S-C. Enhanced oral bioavailability of paclitaxel by co-administration of the $\mathrm{P}$ glycoprotein inhibitor KR30031. Pharm Res 2003; 20:24-30.

81. Swenson ES, Curatolo WJ. Means to enhance penetration. Adv Drug Del Rev 1992; 8:39-92.

82. Jackson MJ. Drug transport across gastrointestinal epithelial. Physiology of the Gastrointestinal Tract. New York: Rave Press; 1987. p. 1597-621.

83. Kim HJ,Yoon KA, Hahn M, Park ES, Chi SC. Preparation and in vitro evaluation of self-microemulsifying drug delivery systems containing idebenone. Drug Dev Ind Pharm 2000; 26:523-9.

84. Asadujjaman MD, Mishuk AU, Novel approaches in lipid based drug delivery systems, Journal of Drug Delivery and Therapeutics, 2013; 3(4):124-130.

85. Meinzer A, Mueller E, Vonderscher J. Microemulsion - a suitable galenical approach for the absorption enhancement of low soluble compounds? B T Gattefosse 1995; 88:21-6.

86. W. C. Pouton, Properties and uses of common formulation lipids, surfactants and co-solvents, in AAPS, Workshop, March 2007.

87. Levy MY, Benita S. Drug release from submicronized o/w emulsion: a new in vitro kinetic evaluation model. Int $\mathbf{J}$ Pharm 1990; 66:29-37.

88. Kommuru TR, Gurley B, Khan MA, Reddy IK. Selfemulsifying drug delivery systems (SEDDS) of coenzyme Q10: formulation development and bioavailability assessment. Int J Pharm 2001; 212:233-46.

GRAS: Generally Recognized as Safe

HLB: hydrophilic-lipophilic balance

MCT: medium-chain triglyceride

LCT: long-chain triglyceride

PEG: polyethylene glycol

TG: Triglyceride

BCS: Biopharmaceutical classification system 